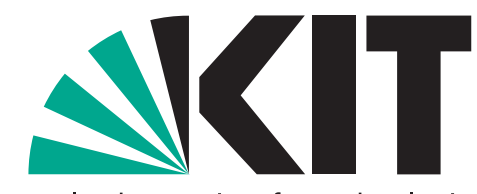

Karlsruher Institut für Technologie

\title{
Time series analysis for financial market meltdowns
}

by Young Shin Kim, Svetlozar T. Rachev, Michele

Leonardo Bianchi, Ivan Mitov, Frank J. Fabozzi

No. 2 | AUGUST 2010

\section{WORKING PAPER SERIES IN ECONOMICS}

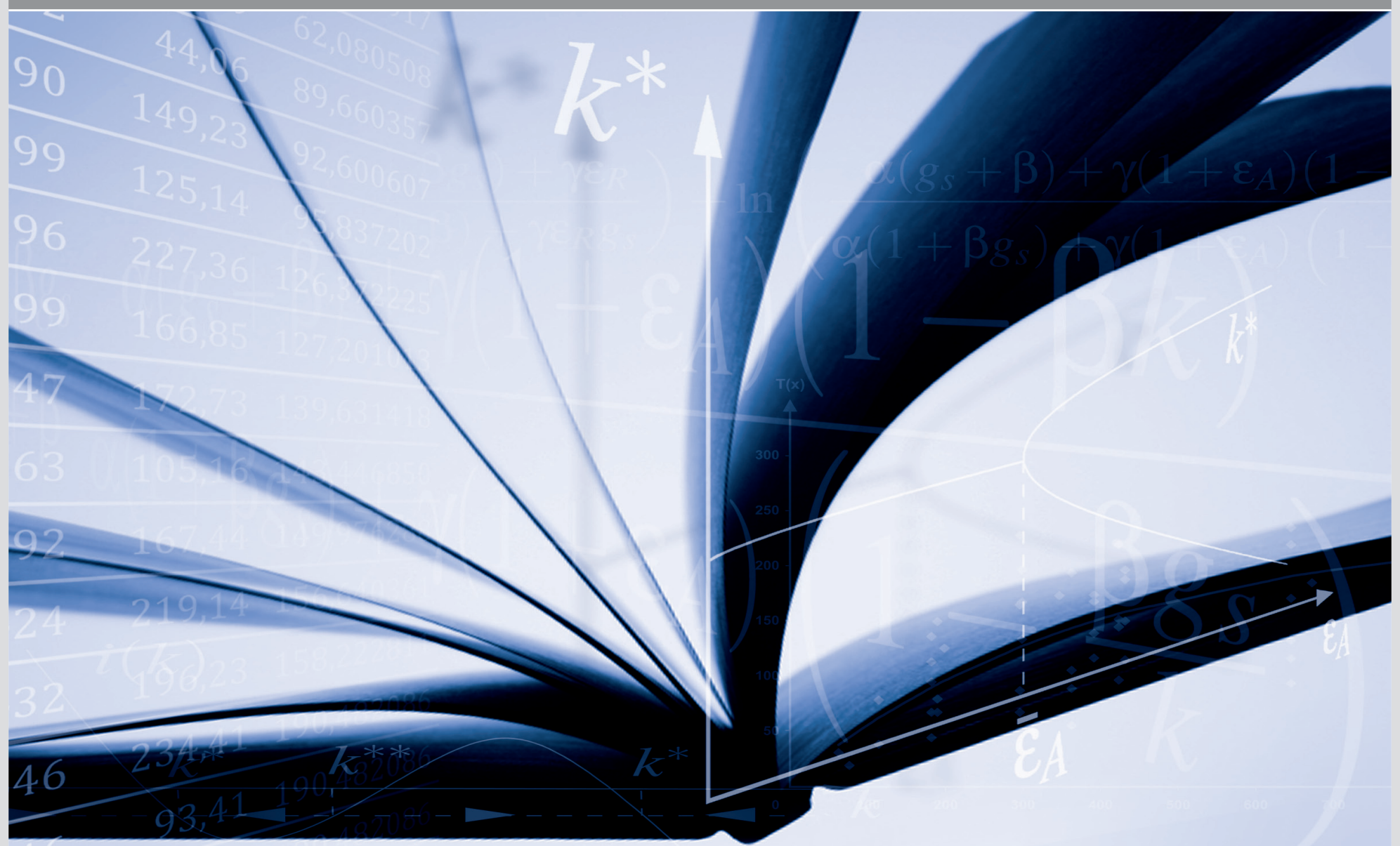




\section{Impressum}

Karlsruher Institut für Technologie (KIT)

Fakultät für Wirtschaftswissenschaften

Institut für Wirtschaftspolitik und Wirtschaftsforschung (IWW)

Institut für Wirtschaftstheorie und Statistik (ETS)

Schlossbezirk 12

76131 Karlsruhe

KIT - Universität des Landes Baden-Württemberg und nationales Forschungszentrum in der Helmholtz-Gemeinschaft

Working Paper Series in Economics

No. 2, August 2010 


\title{
Time Series Analysis for Financial Market Meltdowns
}

\author{
Young Shin Kim ${ }^{\mathrm{a}}$, Svetlozar T. Rachev ${ }^{\mathrm{b}}$, Michele Leonardo Bianchic ${ }^{\mathrm{c}}$, Ivan \\ Mitov ${ }^{\text {, Frank J. Fabozzi }}{ }^{\mathrm{e}}$ \\ ${ }^{a}$ School of Economics and Business Engineering, University of Karlsruhe and KIT. \\ ${ }^{b}$ School of Economics and Business Engineering, University of Karlsruhe and KIT, and \\ Department of Statistics and Applied Probability, University of California, Santa Barbara, and \\ FinAnalytica INC. \\ ${ }^{c}$ Specialized Intermediaries Supervision Department, Bank of Italy. \\ ${ }^{d}$ FinAnalytica INC. \\ ${ }^{e}$ Yale School of Management.
}

\begin{abstract}
There appears to be a consensus that the recent instability in global financial markets may be attributable in part to the failure of financial modeling. More specifically, current risk models have failed to properly assess the risks associated with large adverse stock price behavior. In this paper, we first discuss the limitations of classical time series models for forecasting financial market meltdowns. Then we set forth a framework capable of forecasting both extreme events and highly volatile markets. Based on the empirical evidence presented in this paper, our framework offers an improvement over prevailing models for evaluating stock market risk exposure during distressed market periods.
\end{abstract}

Keywords: ARMA-GARCH model, $\alpha$-stable distribution, tempered stable distribution, value-at-risk (VaR), average value-at-risk (AVaR).

2000 MSC: 60E07, 62M10, 91B28, 91B84

Email addresses: aaron.kim@kit.edu (Young Shin Kim), rachev@kit.edu (Svetlozar T. Rachev), micheleleonardo.bianchi@bancaditalia.it (Michele Leonardo Bianchi), ivan.mitov@finanalytica.com (Ivan Mitov), frank. fabozzi@yale.edu (Frank J. Fabozzi) 


\section{Introduction}

The forecasting of the future behavior of the price of financial instruments is an essential activity in the implementation of risk management and portfolio allocation. The debate between the financial industry and regulators involves whether the sophisticated mathematical and statistical tools that have been employed in risk management and valuation of complex financial instruments have played a role in the recent crisis. In particular, risk measures such as value-at-risk (VaR) and black-box models for assessing the risks that institutional investors and regulated financial entities are exposed to have been singled out as the culprits (see Turner (2009) and Sheedy (2009)). It is within this context that we discuss in this paper a market model that is capable of explaining highly volatile periods. We will demonstrate that the proposed model together with a measure of risk known as the average value-at-risk (AVaR) offers a more reliable risk assessment, particularly during financial crises. Furthermore, we will try to explain how " 25 standard deviation events" in the words of David Viniar, chief financial officer of Goldman Sachs, can occurring. We do so by measuring the probability of occurrence of market crashes by looking at time-series data and showing that this probability strictly depends on the distributional assumption. We then compare these probabilities to the "high standard deviation events" given by the normal probability distribution that is typically assumed.

In order to obtain a good forecast for the distribution of returns, prediction of future market volatility of the market is critical. Most of the recent empirical studies have shown that the amplitude of daily returns varies across time. Moreover, there is ample empirical evidence that if volatility is high, it remains high, and if it is low, it remains low. This means that volatility moves in clusters and for this reason it is important to find a way to explain such observed patterns. This behavior, referred to as "volatility clustering", refers to the tendency of large changes in asset prices (either positive or negative) to be followed by large changes, and small changes to be followed by small changes. The volatility clustering effect can be captured by the autoregressive conditional heteroskedastic $(\mathrm{ARCH})$ and the generalized ARCH (GARCH) models formulated by Engle (1982) and Bollerslev (1986), respectively. However, in this paper we provide empirical evidence that suggests that GARCH models based on the normal distribution would not have performed well in predicting real-world market crashes such as Black Monday (October 19, 1987) and, more recently, the global economic meltdown attributable to the subprime mortgage meltdown in 2007 and the Lehman Brothers failure in the latter half year of 2008. One reason for the poor performance is due to the 
assumption that the innovation of the GARCH model is normally distributed.

Asset management and pricing models require the proper modeling of the return distribution of financial assets. While the return distribution used in the traditional theories of asset pricing such as the capital asset pricing model is the normal distribution, numerous studies that have investigated the empirical behavior of asset returns in financial markets throughout the world reject the hypothesis that asset return distributions are normally distributed. Returns from financial assets show well-defined patterns of leptokurtosis and skewness which cannot be captured by the normality assumption.

Enhanced GARCH models with non-normal innovation distributions have been proposed. For example, Menn and Rachev (2008) used GARCH models with $\alpha$-stable innovations and the smoothly truncated $\alpha$-stable innovations for option pricing. A new class of distributions, the tempered stable distribution, has been proposed recently to deal with the drawbacks of the $\alpha$-stable distribution (see Kim et al. (2008) and Bianchi et al. (2010)).

Most importantly, a suitable measure has to be employed to evaluate market risk. The VaR measure has been adopted as a standard risk measure in the financial industry, having been adopted by regulators to determine the capital requirements for both banking and trading books (see Kiff et al. (2007)). However, the limitations of the VaR measure have been well documented in the academic literature, as well as among regulators and risk managers (see Bookstaber (2009)). Criticisms of this risk measure include (1) a short sample of historical observations is insufficient to assess the risk one-day ahead, (2) the normal distributional assumption is inadequate for forecasting extreme events, and (3) it is difficult to infer future risk from past observed patterns, particularly under stressed scenarios. In this paper, we address these three criticisms by (1) considering a ARMA-GARCH model with non-normal innovation, (2) estimating the model with a sample including 10 years of daily data (including a more realistic measure of risk), principally focused on the negative tail, and (3) backtesting the model during market shocks. By doing so, we hope to provide market participations with more reliable mathematical and statistical tools that can be used to try to understand complex financial market behavior. These tools cannot be used as black-boxes; market players have to understand them to avoid financial debacles.

The risk measure we use in this study is AVaR, which is the average of VaRs less than the VaR for a given tail probability. AVaR, also called conditional value- 
at-risk (CVaR), ${ }^{1}$ is a superior risk measure to VaR because it satisfies all axioms of a coherent risk measure and it is consistent with preference relations of risk-averse investors (see Rachev et al. (2007)). The closed-form solution for AVaR for the $\alpha$-stable distribution, the skewed- $t$ distribution, and the infinitely divisible distributions containing tempered stable distributions have been derived by Stoyanov et al. (2006), Dokov et al. (2008), and Kim et al. (to appear a), respectively.

Hence, in this paper, we discuss autoregressive moving average (ARMA) GARCH models with $\alpha$-stable and tempered stable innovations and then assess the forecasting performance of these models by comparing them to other timeseries models that assume a normal innovation. We empirically test the performance of these models for the S\&P 500 index (SPX) during stressed financial markets. The dataset includes the following stock market crashes: October 1987, October 1997, the turbulent period around the Asian Crisis in 1998 through 1999, the burst of the "dotcom bubble," and the recent subprime mortgage crisis together with the Lehman Brothers failure. We present VaR values for the two indexes for all of these periods. In our backtests of VaR, we evaluate the accuracy of the VaR models. Finally, we present a closed-form solution to the AVaR for the ARMAGARCH model with tempered stable innovations, and compute AVaR values for the two indexes.

The remainder of this paper is organized as follows. ARMA-GARCH models with the $\alpha$-stable and tempered stable innovations are presented in Section 2. In Section 3, we discuss parameter estimation of the ARMA-GARCH models and forecasting return distributions for the two indexes for daily, weekly, and monthly returns. The VaR values and the backtesting of the ARMA-GARCH models with $\alpha$-stable and tempered stable innovations are presented and the results then compared to the classical models such as the equally weighted moving average model and ARMA-GARCH model with normal innovations. The closed-form solution of the AVaR measure for the ARMA-GARCH model with tempered stable innovations is presented in Section 4, together with values of the AVaR for the two indexes. In Section 5, we summarize our principal findings. We briefly review three tempered stable distributions in the appendix.

\section{ARMA-GARCH model with $\alpha$-stable and tempered stable innovations}

Let $\left(S_{t}\right)_{t \geq 0}$ be the asset price process and $\left(y_{t}\right)_{t \geq 0}$ be the the return process of $\left(S_{t}\right)_{t \geq 0}$ defined by $y_{t}=\log \frac{S_{t}}{S_{t-1}}$. We propose the $\operatorname{ARMA}(1,1)-\operatorname{GARCH}(1,1)$

${ }^{1}$ See Pflug (2000) and Rockafellar and Uryasev(2000, 2002). 
model:

$$
\left\{\begin{array}{l}
y_{t}=a y_{t-1}+b \sigma_{t-1} \varepsilon_{t-1}+\sigma_{t} \varepsilon_{t}+c, \\
\sigma_{t}^{2}=\alpha_{0}+\alpha_{1} \sigma_{t-1}^{2} \varepsilon_{t-1}^{2}+\beta_{1} \sigma_{t-1}^{2},
\end{array}\right.
$$

where $\varepsilon_{0}=0$, and a sequence $\left(\varepsilon_{t}\right)_{t \in \mathbb{N}}$ of independent and identically distributed (iid) real random variables. The innovation $\varepsilon_{t}$ is assumed to be the standard normal distribution. In this case, the ARMA $(1,1)-\operatorname{GARCH}(1,1)$ model is referred to as the normal-ARMA-GARCH model.

If $\varepsilon_{t}$ is assumed to be $\alpha$-stable ${ }^{2}$ and tempered stable innovations, then we obtain new ARMA $(1,1)-\operatorname{GARCH}(1,1)$ models. In this paper, we will consider three tempered stable distributions: the standard classical tempered stable (stdCTS), standard modified tempered stable (stdMTS), and standard rapidly decreasing tempered stable (stdRDTS) distributions. New ARMA(1,1)-GARCH(1,1) models with the $\alpha$-stable and three tempered stable innovations are defined as follows:

- Stable-ARMA-GARCH model ${ }^{3}: \varepsilon_{t} \sim S_{\alpha}(\sigma, \beta, \mu)$.

- CTS-ARMA-GARCH model: $\varepsilon_{t} \sim \operatorname{stdCTS}\left(\alpha, \lambda_{+}, \lambda_{-}\right)$.

- RDTS-ARMA-GARCH model: $\varepsilon_{t} \sim \operatorname{stdRDTS}\left(\alpha, \lambda_{+}, \lambda_{-}\right)$.

- MTS-ARMA-GARCH model: $\varepsilon_{t} \sim \operatorname{stdMTS}\left(\alpha, \lambda_{+}, \lambda_{-}\right)$.

Definitions and more details about the three tempered stable distributions are presented in the appendix to this paper.

Substituting $a=0$ and $b=0, \alpha_{1}=0$, and $\beta_{1}=0$ into (1), we obtain the constant volatility $(\mathrm{CV})$ market model. In the $\mathrm{CV}$ model, the conditional variance becomes constant, i.e. $\sigma_{t}=\sqrt{\alpha_{0}}$ for all $t \geq 0$. Substituting $a=0$ and $b=0$ into (1), we obtain the $\operatorname{GARCH}(1,1)$ model. If the innovation distributions are normally distributed, the $\mathrm{CV}$ and $\operatorname{GARCH}(1,1)$ models are referred to as the normal-CV model and the normal-GARCH model, respectively. Similarly, if innovation distributions are $\alpha$-stable distributed, the $\mathrm{CV}$ and $\operatorname{GARCH}(1,1)$ models are referred to as the stable-CV model and the stable-GARCH model, respectively. Moreover, if the innovation distributions are the three tempered-stable distributions, the $\mathrm{CV}$ and $\mathrm{GARCH}(1,1)$ models are referred to as the tempered stable process (e.g., CTS-CV model and CTS-GARCH model).

\footnotetext{
${ }^{2}$ Extensive analysis of $\alpha$-stable distributions and their properties can be found in Samorodnitsky and Taqqu (1994), Rachev and Mittnik (2000), and Stoyanov and Racheva-Iotova (2004a,b).

${ }^{3}$ In subsequent discussions of the $\alpha$-stable distributions in this paper, we restrict ourselves to the non-Gaussian case in which $0<\alpha<2$.
} 
For convenience, the normal time series model refers to the normal-CV, normalGARCH, and normal-ARMA-GARCH models, the stable time series model refers to stable-CV, stable-GARCH, and stable-ARMA-GARCH models, and the tempered stable time series model refers to the $\mathrm{CV}, \operatorname{GARCH}(1,1)$, and $\operatorname{ARMA}(1,1)$ GARCH $(1,1)$ models with the CTS, MTS, and RDTS innovations.

\section{Parameter estimation and forecasting return distribution}

In this section, we estimate the parameters for the $\mathrm{CV}$ model, the $\operatorname{GARCH}(1,1)$ model, and the ARMA $(1,1)-\mathrm{GARCH}(1,1)$ model. We use the historical data of the SPX. We then analyze the probability of market crashes.

Parameters of the CV, GARCH $(1,1)$, and $\operatorname{ARMA}(1,1)-\operatorname{GARCH}(1,1)$ models are estimated from daily, weekly, and monthly returns. For daily returns, we use the closing price of those indexes. Weekly returns are calculated using closing prices on every Friday. If Friday is a holiday, then we use the trading day before that Friday. Monthly returns are calculated using the closing price of the last trading day of each month. In Figure 1, we show the daily, weekly, and monthly returns of the SPX.

Parameters of the normal-CV, normal-GARCH, and normal-ARMA-GARCH models are estimated using the maximum likelihood estimation method (MLE). For the other cases, the parameters are estimated as follows:

1. Estimate parameters $\alpha_{0}, \alpha_{1}, \beta_{1}, a, b, c$ with Student- $t$ distributed innovation by the MLE. For estimating the parameters of the CV models, all parameters except $\alpha_{0}$ are assumed to be zero. For the $\operatorname{GARCH}(1,1)$ model, the parameters $a$ and $b$ are assumed to be zero.

2. Extract residuals using the estimated parameters.

3. Fit the parameters of the innovation distribution (the $\alpha$-stable, CTS, MTS, and RDTS distributions) to the extracted residuals using the MLE. ${ }^{4}$

\subsection{Daily return of $S \& P 500$ index}

We selected five days of market crashes for daily returns of the SPX: October 19, 1987 (Black-Monday), October 27, 1997 (Asian Turmoil), August 31, 1998 (Russian Default), April 14, 2000 (Dotcom Collapse), and September 29, 2008

\footnotetext{
${ }^{4}$ See Bianchi et al. (to appear).
} 
(U.S. financial crisis). In order to investigate the forecasting performance of each model, we estimate parameters using 10 years of historical data through the closest trading day before the selected crash day. The returns and data for estimating the parameters are described in Table 1.

In Tables 3 to 7 we report estimation results for each dataset described in Table 1. These tables provide parameter estimation results for the $\mathrm{CV}, \operatorname{GARCH}(1,1)$, and ARMA(1,1)-GARCH $(1,1)$ models with the normal and Student- $t$ distributed innovations as well as estimated parameters for the $\alpha$-stable, standard CTS, standard MTS, and standard RDTS distributions. For the assessment of the goodnessof-fit, we use the Kolmogorov-Smirnov (KS) test and also calculate the AndersonDarling (AD) statistic, the latter statistic providing a better test to evaluate the tail fit.

Based on the goodness-of-fit statistics reported in Tables 3 to 7, we conclude the following:

- For all six datasets, the three time series models based on the normal distribution are rejected by the KS test at the $1 \%$ significance level.

- The stable-CV model is rejected by the KS test at the $1 \%$ significance level in the U.S. financial crisis case.

- The KS test applied to the data ending with September 26, 2008, the day before the biggest loss during the U.S. financial crisis, shows that the CTS-CV, the MTS-CV, the RDTS-CV, the RDTS-GARCH, and the RDTS-ARMAGARCH models are not rejected while all other models are rejected at the $1 \%$ significance level.

- The AD statistic for the three time series models based on the normal distribution are significantly larger than those of the other models. That means the normal distribution cannot describe the fat-tail behavior of the empirical innovation distribution.

Based on the estimated parameters, we can calculate the probabilities of occurrence of those crashes. The average times of occurrence of crashes are calculated by

$$
\frac{1}{250 \cdot P\left[\varepsilon_{t} \leq \varepsilon_{t}^{*}\right]},
$$

where $\varepsilon_{t}^{*}$ is observed residual at time $t$. By taking into consideration the estimates of the above selected models, in Table 3 we provide the residuals observed the 
day before the crash, and the probabilities that the Black Monday collapse will happen, together with the average time of occurrence as defined in (2).

From the results reported in Table 3, we see that the probabilities that the Black Monday decline will happen based on the estimated three normal time series models are considerably less than the other models. At the same time, average time of occurrence based on the three normal time series models are significantly long. That is, the crash should happen only once every $7.966 \cdot 10^{141}$ years under the normal-CV model. If we use the normal-GARCH and normal-ARMA-GARCH models, the crash is expected to happen once every $2.554 \cdot 10^{39}$, and $2.904 \cdot 10^{39}$ years, respectively. Those time values are longer than the age of universe. ${ }^{5}$ In reality, a similar fall should be expected once every 30-50 years, and hence those three models based on the normal distributional assumption are not realistic. In contrast, the three models based on the $\alpha$-stable distribution are more realistic than the three models based on the normal one. Average times of occurrence are 80 years, 37.26 years, and 40.31 years for the stable-CV, stable-GARCH, and stableARMA-GARCH models, respectively. The nine models that consider tempered stable distributions have better performance than the corresponding three normal time series models. The nine tempered stable time series models have longer average times of occurrence than the corresponding stable time series model, but significantly shorter than those of the corresponding normal time series models.

In Tables 4 to 6 we present the empirical analysis conducted for the Asian Turmoil (Table 4), the Russian Default (Table 5), and the Dotcom Collapse (Table 6). In these investigations, we obtain similar results as in the Black Monday case. That is, the normal time series models are not realistic. Average times to occurrence of the crash for the three stable time series models are shorter than the other models investigated.

We also investigate the probability that the crash on September 29, 2008 would occur. On that day the SPX declined 9\%. This crisis was caused by the default of Lehman Brothers on September 15, 2008, and the market at that time was characterized by increased volatility. We estimate parameters using the data until September 26, 2008 and forecast the probability that the crash on September 29, 2008 will occur. Average times of occurrences are $1.99 \cdot 10^{13}, 502.1$, and 732.3 years for the normal-CV, normal-GARCH, and normal-ARMA-GARCH models, respectively. That is, we do not obtain any serious alerts from the three normal

\footnotetext{
${ }^{5}$ Astronomers estimated that the age of universe is between 12 and 14 billion years. See http://map.gsfc.nasa.gov/.
} 
time series models even though the models do consider the volatility clustering effect. Constant volatility models such as the CTS-CV, MTS-CV, and RDTS-CV models do not perform satisfactorily either. In contrast, the $\alpha$-stable and tempered stable time series models that account for volatility clustering have better forecasting power. Those models provide very short times of occurrence for the crisis, and hence one can consider them to be superior early-warning models.

\subsection{Weekly and monthly returns of $S \& P 500$ index}

Next, we selected three of the market crashes analyzed in Section 3.1. However, instead of considering daily returns, we analyze the time series of SPX weekly returns ending in the week October 19-23, 1987 (Black Monday), April 10-14, 2000 (Dotcom Collapse), and October 6-10, 2008 (U.S. financial crisis). In order to investigate the forecasting performance of each model, we estimate parameters for historical weekly returns one week before the selected crash days. Then, we also analyze the time series of SPX monthly returns, selecting the months October 1987 and October 2008. The SPX dropped $24.54 \%$, and $18.42 \%$ in October 1987 and October 2008, respectively. Table 2 describes returns and data considered in the estimation.

Tables 8 to 12 report the estimated parameters of the $\mathrm{CV}$, GARCH, and ARMAGARCH models with normal and Student- $t$ distributed innovations, the estimates relative to $\alpha$-stable, standard CTS, standard MTS, and standard RDTS distributions, the KS statistics with the corresponding $p$-values, and the AD statistics for all the models considered, for weekly and monthly returns. These results indicate the following:

- For Black Monday and the U.S. financial crisis, the normal-CV and the stable-ARMA-GARCH models are rejected by the KS test at the $1 \%$ significance level.

- In all cases, the stable-CV model is rejected by the KS test at the $1 \%$ significance level.

- In the U.S. financial crisis case, the stable-GARCH model is rejected by the $\mathrm{KS}$ test at the $1 \%$ significance level.

- The three stable and nine tempered stable time series models have a better AD statistic than those of the three normal time series models.

- The nine tempered stable time series models are not rejected by the KS test for all datasets. 
Based on Tables 11 and 12, we conclude the following:

- The KS test does not reject any time series model at the $1 \%$ significance level.

- The three normal time series models have significantly larger AD statistics than the other models. That is, the normal time series models cannot explain the tail properties of the monthly return distribution better than the other models investigated.

Based on the estimated parameters, we can calculate the probability that those crashes will happen. In Tables 8 to 10 we also provide observed residuals for weekly returns and probabilities that the collapse will happen in weekly analysis. In Tables 11 and 12 we provide the observed residuals for monthly returns, as well as the probabilities that the collapse will happen in monthly analysis. In the weekly analysis, the average time of occurrence is calculated by

$$
\frac{1}{53 \cdot P\left[\varepsilon_{t} \leq \varepsilon_{t}^{*}\right]},
$$

where $\varepsilon_{t}^{*}$ is observed residual of weekly returns, while in the monthly analysis, it is calculated by

$$
\frac{1}{12 \cdot P\left[\varepsilon_{t} \leq \varepsilon_{t}^{*}\right]},
$$

where $\varepsilon_{t}^{*}$ is observed residual for monthly returns.

Following are fair conclusions from the results reported in the tables:

- Average time of occurrences of the three normal time series models are very large and unrealistic in both the weekly analysis and the monthly analysis.

- The three stable time series models are more realistic than the three normal time series models in view of average time of occurrences.

- In ARMA-GARCH models, average times of occurrence of the stable-ARMA-GARCH models for both the weekly analysis and the monthly analysis are shorter than the other models investigated.

- Average times to occurrences of the CTS-ARMA-GARCH, MTS-ARMAGARCH, and RDTS-ARMA-GARCH are longer than the three normalARMA-GARCH models and shorter than the three stable-ARMA-GARCH models for the weekly and monthly cases, respectively. 
- In the CV and GARCH models, we obtain the same conclusion as the ARMA-GARCH model cases.

\section{VaR and AVaR for ARMA-GARCH model}

In this section we discuss the VaR and AVaR for the ARMA-GARCH model with stable and tempered stable innovations.

\subsection{VaR and backtesting}

The definition of VaR with the significance level $\eta$ is

$$
\operatorname{VaR}_{\eta}(X)=-\inf \{x \in \mathbb{R} \mid P(X \leq x)>\eta\} .
$$

Considering the ARMA-GARCH model defined, we can define the VaR for the information until time $t$ with significance level $\eta$ as

$$
\operatorname{VaR}_{t, \eta}\left(y_{t+1}\right)=-\inf \left\{x \in \mathbb{R} \mid P_{t}\left(y_{t+1} \leq x\right)>\eta\right\},
$$

where $P_{t}(A)$ is the conditional probability of a given event $A$ for the information until time $t$.

We consider five models: the normal-CV, the exponentially weighted moving average (EWMA), ${ }^{6}$ the normal-ARMA-GARCH, the stable-ARMA-GARCH, and the CTS-ARMA-GARCH. For all five models we estimate the parameters by considering the time series from December 14, 2004 to December 31, 2008. For each daily estimation, we used about 10 years of historical return data for the SPX. We then computed VaRs for the five models. Figure 2 shows the SPX daily returns and negative values of daily VaRs with $1 \%$ significance level (i.e. $\left.-\operatorname{VaR}_{t, 0.01}\left(y_{t+1}\right)\right)$ for all five models considered. Based on those figures, we observe that the normal-CV model is not able to capture market crashes.

For evaluating the accuracy of VaR for the five models, we perform the backtesting by Kupiec's proportion of failures test developed by Kupiec (1995). For this, the number of violations (violations occur when the actual loss exceeds the estimate) from the empirical data are compared to the accepted number of exceedances at a given significant level. In Table 13 we report the number of violations and $p$-values of Kupiec's backtest for the SPX. We count the number of

\footnotetext{
${ }^{6}$ We follow the EWMA model of J.P. Morgan RiskMetrics. In the model, the daily volatility formula is given by $\sigma_{t}^{2}=\lambda \sigma_{t-1}^{2}+(1-\lambda) y_{t-1}^{2}$ with $\lambda=0.94$.
} 
violations and the corresponding $p$-values for $1 \%$-VaRs for the five models by considering different time periods: 1,2 , and 4 years.

From Table 13, we conclude the following for the SPX:

- All five models are not rejected at the significance level of $5 \%$ in the period from (1) December 14, 2004 to December 15, 2005 and from December 16, 2005 to December 20, 2006 (1-year backtest), and (2) December 14, 2004 to December 20, 2006 (2-year backtest).

- For all crash dates investigated, the stable-ARMA-GARCH model is not rejected at the significance level of $5 \%$, and the CTS-ARMA-GARCH model is not rejected at the significance level of $1 \%$.

- The normal-CV, EWMA, normal-ARMA-GARCH models are rejected, but the stable-ARMA-GARCH and CTS-ARMA-GARCH models are not rejected at the significance level of $5 \%$ for the period from December 21 , 2006 to December 31, 2008 (2-year backtest) and December 14, 2004 to December 31, 2008 (4-year backtest).

According to the 1-year backtest, the discrepancy between the normal-CV, EWMA, normal-ARMA-GARCH, and stable and CTS-ARMA-GARCH is observed one year prior the crash of 2008. Hence, we consider that discrepancy as early warning indicators of a forthcoming market crash.

\subsection{AVaR for the ARMA-GARCH model with tempered stable innovations}

In this section, we discuss AVaR for the ARMA-GARCH model with CTS innovation, and provide an empirical example of the AVaR under the CTS-ARMAGARCH model for the SPX.

The definition of AVaR with the significance level $\eta$ is

$$
\operatorname{AVaR}_{\eta}(X)=\frac{1}{\eta} \int_{0}^{\eta} \operatorname{VaR}_{\epsilon}(X) d \epsilon
$$

where $\operatorname{VaR}_{\epsilon}(X)$ is the $\operatorname{VaR}$ of $X$ with the significance level $\epsilon$. If the distribution of $X$ is continuous, then we have

$$
A \operatorname{VaR}_{\eta}(X)=-E\left[X \mid X<-\operatorname{VaR}_{\eta}(X)\right] .
$$

Consider the ARMA-GARCH model defined in (1). Since for every $t>0$ the distribution of $y_{t}$ is continuous, we define the conditional AVaR of $y_{t+1}$ for the 
information until time $t$ with the significance level $\eta$ by the following form:

$$
A \operatorname{Va} R_{t, \eta}\left(y_{t+1}\right)=-E_{t}\left[y_{t+1} \mid y_{t+1}<-V a R_{t, \eta}\left(y_{t+1}\right)\right],
$$

where $\operatorname{VaR} R_{t, \eta}\left(y_{t+1}\right)$ is the $\mathrm{VaR}$ of $y_{t+1}$ for the information until time $t$ with the significance level $\eta$ and $E_{t}$ is the conditional expectation for the information until time $t$. By equation (1), we have

$$
\begin{aligned}
& A V a R_{t, \eta}\left(y_{t+1}\right) \\
& =-E_{t}\left[a y_{t}+b \sigma_{t} \varepsilon_{t}+\sigma_{t+1} \varepsilon_{t+1}+c \mid y_{t+1}<-V a R_{t, \eta}\left(y_{t+1}\right)\right] .
\end{aligned}
$$

Since $y_{t}, \sigma_{t}, \varepsilon_{t}$, and $\sigma_{t+1}$ are determined at time $t$, we have

$$
\begin{aligned}
& A V a R_{t, \eta}\left(y_{t+1}\right) \\
& =-\left(c+a y_{t}+b \sigma_{t} \varepsilon_{t}+\sigma_{t+1} E_{t}\left[\varepsilon_{t+1} \mid y_{t+1}<-\operatorname{VaR} R_{t, \eta}\left(y_{t+1}\right)\right]\right) .
\end{aligned}
$$

Moreover, since we can prove that

$$
-V a R_{t, \eta}\left(y_{t+1}\right)=a y_{t}+b \sigma_{t} \varepsilon_{t}+\sigma_{t+1}\left(-V a R_{t, \eta}\left(\varepsilon_{t+1}\right)\right)+c,
$$

we obtain

$$
A V a R_{t, \eta}\left(y_{t+1}\right)=-\left(c+a y_{t}+b \sigma_{t} \varepsilon_{t}+\sigma_{t+1} E_{t}\left[\varepsilon_{t+1} \mid \varepsilon_{t+1}<-V a R_{t, \eta}\left(\varepsilon_{t+1}\right)\right]\right),
$$

or

$$
A V a R_{t, \eta}\left(y_{t+1}\right)=-\left(c+a y_{t}+b \sigma_{t} \varepsilon_{t}\right)+\sigma_{t+1} A V a R_{t, \eta}\left(\varepsilon_{t+1}\right) .
$$

Since $\varepsilon_{t+1}$ is independent of the information until time $t$ and it is continuously distributed, we have

$$
A \operatorname{VaR} R_{t, \eta}\left(\varepsilon_{t+1}\right)=-E\left[\varepsilon_{t+1} \mid \varepsilon_{t+1}<-\operatorname{VaR}_{\eta}\left(\varepsilon_{t+1}\right)\right]=\operatorname{AVa} R_{\eta}\left(\varepsilon_{t+1}\right) .
$$

If $\varepsilon_{t+1}$ is tempered stable, then the formula $A V a R_{t, \eta}\left(\varepsilon_{t+1}\right)$ can be obtained by the following proposition (see Kim et al. (to appear a)). ${ }^{7}$

Proposition Let $Y$ be a random variable for the return of an asset or portfolio.

\footnotetext{
${ }^{7} \mathrm{AVaR}$ is defined on the loss distribution in Kim et al. (to appear a). We changed the proposition because we are looking at the return distribution.
} 
Suppose $Y$ is infinitely divisible and the distribution function of $Y$ is continuous. If there is $\rho>0$ such that $\left|\phi_{Y}(-u+i \rho)\right|<\infty$ for all $u \in \mathbb{R}$, then

$$
\begin{aligned}
& \operatorname{AVaR}_{\eta}(Y) \\
& =\operatorname{VaR}_{\eta}(Y)-\frac{e^{-\operatorname{VaR}_{\eta}(Y) \rho}}{\pi \eta} \Re\left(\int_{0}^{\infty} e^{-i u \operatorname{VaR}_{\eta}(Y)} \frac{\phi_{Y}(-u+i \rho)}{(-u+i \rho)^{2}} d u\right) .
\end{aligned}
$$

In Figure 3, we report the SPX daily returns and daily values of $\left(-\mathrm{AVaR}_{t, 0.01}\right.$ $\left.\left(y_{t+1}\right)\right)$ for the normal-ARMA-GARCH and CTS-ARMA-GARCH models. Comparing Figure 3 to Figure 2, one can graphically check the differences between these two risk measures. In particular, AVaR seems to be more conservative under stressed scenarios. This means that AVaR can be considered a good indicator during highly volatile markets.

In Figure 4, we present the daily differences for the $\mathrm{S} \& \mathrm{P} 500$ between $\mathrm{AVaR}_{t, 0.01}$ $\left(y_{t+1}\right)$ for the CTS-ARMA-GARCH model and $\operatorname{VaR}_{t, 0.01}\left(y_{t+1}\right)$ for the normalARMA-GARCH model. The spreads from July 2007 to September 2008 are typically larger than the spreads until July 2007. Finally, the index declines sharply in September 2008. The same phenomenon is observed for the the daily differences of $\mathrm{AVaR}_{t, 0.01}\left(y_{t+1}\right)$ between the normal-ARMA-GARCH model and CTSARMA-GARCH model presented in Figure 5. Hence, we consider the increasing spread between 1\%-AVaR for the CTS-ARMA-GARCH model and 1\%-VaR (or $1 \% \mathrm{AVaR}$ ) for the normal-ARMA-GARCH model and as early warning indicators of a pending market crash.

\section{Conclusion}

In this paper, we discussed models with stable and tempered stable innovations, and provided an assessment of their forecasting power relative to other models widely used in the industry. The proposed models are applied to the analysis of the S\&P 500 index during highly volatile markets.

Our first finding is that the time series models based on the assumption of a normal innovation do not provide a reliable forecast of the future distribution of returns, even if they account for volatility clustering. In particular, we our empirical evidence indicates that time series models with stable and tempered stable innovations have better predictable power in measuring market risk compared to standard models based on the normal distribution assumption.

We also analyzed the behavior of VaR depending on different distributional assumption. We backtested VaR by considering the last four years of log returns 
for the SPX. The Kupiec's proportion of failures test rejects the three normal time series models considered, but does not reject the stable and tempered stable time series models.

Finally, we derived a closed-form solution for the AVaR for the ARMA-GARCH models with tempered stable innovation and applied this formula to calculate daily AVaRs with the CTS-ARMA-GARCH models with respect to the last four years of data.

The principal findings of the paper are twofold. First, in a low volatile market, the models proposed in this paper are practically identical to the corresponding Gaussian models. Second, the proposed models can be used as early warning systems for a forthcoming sharp market downturn.

\section{Acknowledgement}

Rachev gratefully acknowledges research support through grants from the Division of Mathematical, Life and Physical Sciences, College of Letters and Science, University of California, Santa Barbara, the Deutschen Forschungsgemeinschaft, and the Deutscher Akademischer Austausch Dienst. Bianchi acknowledges that the views expressed in this paper are his alone and should not be attributed to those of his employer.

\section{References}

Bianchi, M. L., Rachev, S. T., Kim, Y. S., Fabozzi, F. J., 2010. Tempered infinitely divisible distributions and processes. Theory of Probability and Its Applications (TVP), Society for Industrial and Applied Mathematics (SIAM).

Bianchi, M. L., Rachev, S. T., Kim, Y. S., Fabozzi, F. J., to appear. Tempered stable distributions and processes in finance: numerical analysis. In: Corazza, M., Pizzi, C. (Eds.), Mathematical and Statistical Methods for Actuarial Sciences and Finance - MAF 2008.

Bollerslev, T., 1986. Generalized autoregressive conditional heteroskedasticity. Journal of Econometrics 31, 307-327.

Bookstaber, R., September 2009. The risks of financial modeling: VaR and the economic meltdown. Committee on Science and Technology, United States House of Representatives. 
Boyarchenko, S. I., Levendorskiï, S. Z., 2000. Option pricing for truncated Lévy processes. International Journal of Theoretical and Applied Finance 3, 549552.

Carr, P., Geman, H., Madan, D., Yor, M., 2002. The fine structure of asset returns: An empirical investigation. Journal of Business 75 (2), 305-332.

Dokov, S., Stoyanov, S. V., Rachev, S. T., 2008. Computing VaR and AVaR of skewed-t distribution. Journal of Applied Functional Analysis 3 (1), 189-208.

Engle, R., 1982. Autoregressive conditional heteroskedasticity with estimates of the variance of united kingdom inflation. Econometrica 50, 987-1007.

Kiff, J., Kodres, L., Klueh, U., Mills, P., 2007. Do market risk management techniques amplify systemic risks? In: Global Financial Stability Report, Financial Market Turbulence Causes, Consequences, and Policies. World Economic and Financial Surveys. International Monetary Fund, pp. October, 52-76.

Kim, Y. S., 2005. The modified tempered stable processes with application to finance, ph.D thesis, Sogang University.

Kim, Y. S., Rachev, S. T., , Bianchi, M. L., Fabozzi, F. J., to appear a. Computing VaR and AVaR in infinitely divisible distributions. Probability and Mathematical Statistics.

Kim, Y. S., Rachev, S. T., Bianchi, M. L., Fabozzi, F. J., 2008. Financial market models with Lévy processes and time-varying volatility. Journal of Banking and Finance 32, 1363-1378.

Kim, Y. S., Rachev, S. T., Bianchi, M. L., Fabozzi, F. J., to appear b. Tempered stable and tempered infinitely divisible GARCH models. Journal of Banking and Finance.

Kim, Y. S., Rachev, S. T., Chung, D. M., Bianchi, M. L., 2009. The modified tempered stable distribution, GARCH-models and option pricing. Probability and Mathematical Statistics 29 (1), 91-117.

Koponen, I., 1995. Analytic approach to the problem of convergence of truncated Lévy flights towards the Gaussian stochastic process. Physical Review E 52, 1197-1199. 
Kupiec, P., 1995. Techniques for verifying the accuracy of risk measurement models. Journal of Derivatives 6, 6-24.

Menn, C., Rachev, S. T., 2008. Smoothly truncated stable distributions, GARCHmodels, and option pricing. Mathematical Methods in Operation ResearchTo appear.

Pflug, G., 2000. Some remarks on the value-at-risk and the conditional value-atrisk. In: Uryasev, S. (Ed.), Probabilistic Constrained Optimization: Methodology and Applications. Kluwer Academic Publishers, pp. 272-281.

Rachev, S. T., Mittnik, S., 2000. Stable Paretian Models in Finance. John Wiley \& Sons, New York.

Rachev, S. T., Stoyanov, S., Fabozzi, F. J., 2007. Advanced Stochastic Models, Risk Assessment, and Portfolio Optimization: The Ideal Risk, Uncertainty, and Performance Measures. John Wiley\&Sons, Hoboken, New Jersey.

Rockafellar, R. T., Uryasev, S., 2000. Optimization of conditional value-at-risk. The Journal of Risk 2 (3), 21-41.

Rockafellar, R. T., Uryasev, S., 2002. Conditional value-at-risk for general loss distributions. Journal of Banking \& Finance 26, 1443-1471.

Samorodnitsky, G., Taqqu, M. S., 1994. Stable non-Gaussian random processes. Chapman \& Hall/CRC.

Sheedy, E., 2009. It's NOT the econometrics, stupid. Risk Magazine, September.

Stoyanov, S., Racheva-Iotova, B., 2004a. Univariate stable laws in the field of finance. approximation of density and distribution functions. Journal of Concrete and Applicable Mathematics 2 (1), 37-58.

Stoyanov, S., Racheva-Iotova, B., 2004b. Univariate stable laws in the field of finance. parameter estimation. Journal of Concrete and Applicable Mathematics 2 (4), 24-49.

Stoyanov, S., Samorodnitsky, G., Rachev, S. T., Ortobelli, S., 2006. Computing the portfolio conditional value-at-risk in the $\alpha$-stable case. Probability and Mathematical Statistics 26 (1), 1-22. 
Turner, A., March 2009. The Turner Review: A regulatory response to the global banking crisis. FSA.

\section{Appendix}

In this section we review three classes of tempered stable distributions for modeling a return distribution. Generally, these distributions do not have closedform solutions for their probability density function. Instead, they are defined by their characteristic function.

Let $\alpha \in(0,2) \backslash\{1\}, C, \lambda_{+}, \lambda_{-}>0$, and $m \in \mathbb{R}$.

1. A random variable $X$ is said to follow the classical tempered stable (CTS) distribution if the characteristic function of $\mathrm{X}$ is given by

$$
\begin{aligned}
\phi_{X}(u)= & \phi_{C T S}\left(u ; \alpha, C, \lambda_{+}, \lambda_{-}, m\right) \\
= & \exp \left(i u m-i u C \Gamma(1-\alpha)\left(\lambda_{+}^{\alpha-1}-\lambda_{-}^{\alpha-1}\right)\right. \\
& \left.\quad+C \Gamma(-\alpha)\left(\left(\lambda_{+}-i u\right)^{\alpha}-\lambda_{+}^{\alpha}+\left(\lambda_{-}+i u\right)^{\alpha}-\lambda_{-}^{\alpha}\right)\right),
\end{aligned}
$$

and we denote $X \sim \operatorname{CTS}\left(\alpha, C, \lambda_{+}, \lambda_{-}, m\right){ }^{8}$

2. A random variable $X$ is said to follow the modified tempered stable (MTS) distribution ${ }^{9}$ if the characteristic function of $X$ is given by

$$
\begin{aligned}
\phi_{X}(u)= & \phi_{M T S}\left(u ; \alpha, C, \lambda_{+}, \lambda_{-}, m\right) \\
= & \exp \left(i u m+C\left(G_{R}\left(u ; \alpha, \lambda_{+}\right)+G_{R}\left(u ; \alpha, \lambda_{-}\right)\right)\right. \\
& \left.+i u C\left(G_{I}\left(u ; \alpha, \lambda_{+}\right)-G_{I}\left(u ; \alpha, \lambda_{-}\right)\right)\right),
\end{aligned}
$$

where for $u \in \mathbb{R}$,

$$
G_{R}(x ; \alpha, \lambda)=2^{-\frac{\alpha+3}{2}} \sqrt{\pi} \Gamma\left(-\frac{\alpha}{2}\right)\left(\left(\lambda^{2}+x^{2}\right)^{\frac{\alpha}{2}}-\lambda^{\alpha}\right)
$$

\footnotetext{
${ }^{8}$ The CTS distribution has been introduced under different names including: truncated Lévy flight by Koponen (1995), the KoBoL distribution by Boyarchenko and Levendorskii (2000), and the CGMY distribution by Carr et al. (2002).

${ }^{9}$ See Kim (2005) and Kim et al. (2009).
} 
and

$$
G_{I}(x ; \alpha, \lambda)=2^{-\frac{\alpha+1}{2}} \Gamma\left(\frac{1-\alpha}{2}\right) \lambda^{\alpha-1}\left[{ }_{2} F_{1}\left(1, \frac{1-\alpha}{2} ; \frac{3}{2} ;-\frac{x^{2}}{\lambda^{2}}\right)-1\right],
$$

where ${ }_{2} F_{1}$ is the hypergeometric function. We denote an MTS distributed random variable $X$ by $X \sim \operatorname{MTS}\left(\alpha, C, \lambda_{+}, \lambda_{-}, m\right)$.

3. A random variable $X$ is said to follow the rapidly decreasing tempered stable (RDTS) distribution ${ }^{10}$ if the characteristic function of $X$ is given by

$$
\begin{aligned}
\phi_{X}(u) & =\phi_{R D T S}\left(u ; \alpha, C, \lambda_{+}, \lambda_{-}, m\right) \\
& =\exp \left(i u m+C\left(G\left(i u ; \alpha, \lambda_{+}\right)+G\left(-i u ; \alpha, \lambda_{-}\right)\right)\right),
\end{aligned}
$$

where

$$
\begin{aligned}
G(x ; \alpha, \lambda)= & 2^{-\frac{\alpha}{2}-1} \lambda^{\alpha} \Gamma\left(-\frac{\alpha}{2}\right)\left(M\left(-\frac{\alpha}{2}, \frac{1}{2} ; \frac{x^{2}}{2 \lambda^{2}}\right)-1\right) \\
& +2^{-\frac{\alpha}{2}-\frac{1}{2}} \lambda^{\alpha-1} x \Gamma\left(\frac{1-\alpha}{2}\right)\left(M\left(\frac{1-\alpha}{2}, \frac{3}{2} ; \frac{x^{2}}{2 \lambda^{2}}\right)-1\right),
\end{aligned}
$$

and $M$ is the confluent hypergeometric function. In this case, we denote $X \sim \operatorname{RDTS}\left(\alpha, C, \lambda_{+}, \lambda_{-}, m\right)$.

The cumulants of $X$ are defined by

$$
c_{n}(X)=\left.\frac{\partial^{n}}{\partial u^{n}} \log E\left[e^{i u X}\right]\right|_{u=0}, \quad n=1,2,3, \cdots .
$$

For the three tempered stable distributions, we have $E[X]=c_{1}(X)=m$. The cumulants of the three tempered stable distributions for $n=2,3, \cdots$ are presented in the following table:

\begin{tabular}{cc} 
Distribution of $X$ & $c_{n}(X)$ for $n=2,3, \cdots$ \\
\hline CTS & $C \Gamma(n-\alpha)\left(\lambda_{+}^{\alpha-n}+(-1)^{n} \lambda_{-}^{\alpha-n}\right)$ \\
MTS & $2^{n-\frac{\alpha+3}{2}} C \Gamma\left(\frac{n+1}{2}\right) \Gamma\left(\frac{n-\alpha}{2}\right)\left(\lambda_{+}^{\alpha-n}+(-1)^{n} \lambda_{-}^{\alpha-n}\right)$ \\
RDTS & $2^{\frac{n-\alpha-2}{2}} C \Gamma\left(\frac{n-\alpha}{2}\right)\left(\lambda_{+}^{\alpha-n}+(-1)^{n} \lambda_{-}^{\alpha-n}\right)$ \\
\hline
\end{tabular}

\footnotetext{
${ }^{10}$ See Kim et al. (to appear b).
} 
By substituting the appropriate value for the two parameters $m$ and $C$ into the three tempered stable distributions, we can obtain tempered stable distributions with zero mean and unit variance. That is,

1. $X \sim \operatorname{CTS}\left(\alpha, C, \lambda_{+}, \lambda_{-}, 0\right)$ has zero mean and unit variance by substituting

$$
C=\left(\Gamma(2-\alpha)\left(\lambda_{+}^{\alpha-2}+\lambda_{-}^{\alpha-2}\right)\right)^{-1}
$$

The random variable $X$ is referred to as the standard CTS distribution with parameters $\left(\alpha, \lambda_{+}, \lambda_{-}\right)$and denoted by $X \sim \operatorname{stdCTS}\left(\alpha, \lambda_{+}, \lambda_{-}\right)$.

2. $X \sim \operatorname{MTS}\left(\alpha, C, \lambda_{+}, \lambda_{-}, 0\right)$ has zero mean and unit variance by substituting

$$
C=2^{\frac{\alpha+1}{2}}\left(\sqrt{\pi} \Gamma\left(1-\frac{\alpha}{2}\right)\left(\lambda_{+}^{\alpha-2}+\lambda_{-}^{\alpha-2}\right)\right)^{-1}
$$

The random variable $X$ is referred to as the standard MTS distribution and denoted by $X \sim \operatorname{stdMTS}\left(\alpha, \lambda_{+}, \lambda_{-}\right)$.

3. $X \sim \operatorname{RDTS}\left(\alpha, C, \lambda_{+}, \lambda_{-}, 0\right)$ has zero mean and unit variance by substituting

$$
C=2^{\frac{\alpha}{2}}\left(\Gamma\left(1-\frac{\alpha}{2}\right)\left(\lambda_{+}^{\alpha-2}+\lambda_{-}^{\alpha-2}\right)\right)^{-1}
$$

The random variable $X$ is referred to as the standard RDTS distribution and denoted by $X \sim \operatorname{stdRDTS}\left(\alpha, \lambda_{+}, \lambda_{-}\right)$. 
Table 1: Data for analyzing daily return of the SPX

\begin{tabular}{ccccc} 
Crash & Date & Return & Data for forecasting & $\begin{array}{c}\text { Number of } \\
\text { observations }\end{array}$ \\
\hline Black Monday & Oct. 19,1987 & $-23 \%$ & $\begin{array}{l}\text { daily return ending } \\
\text { with Oct. 16, 1987 }\end{array}$ & 2,490 \\
Asian Turmoil & Oct. 27,1997 & $-7 \%$ & $\begin{array}{l}\text { daily return ending } \\
\text { with Oct. 24, 1997 }\end{array}$ & 2,504 \\
Russian Default & Aug. 31,1998 & $-7 \%$ & $\begin{array}{l}\text { daily return ending } \\
\text { with Aug. 28, 1998 }\end{array}$ & 2,503 \\
Dotcom Collapse & Apr. 14,2000 & $-6 \%$ & $\begin{array}{l}\text { daily return ending } \\
\text { with Apr. 13, 2000 } \\
\text { daily return ending } \\
\text { with Sept. 26, 2008 }\end{array}$ & 2,504 \\
U.S. Financial Crisis & Sept. 29,2008 & $-9 \%$ & \\
\hline
\end{tabular}

Table 2: Data for analyzing weekly and monthly returns of the SPX

Data for weekly returns

\begin{tabular}{|c|c|c|c|c|}
\hline Crash & Date & Return & Data for forecasting & $\begin{array}{c}\text { Number of } \\
\text { observations }\end{array}$ \\
\hline Black Monday & Oct. $19-23,1987$ & $-13.01 \%$ & $\begin{array}{l}\text { weekly return ending } \\
\text { with Oct. } 16,1987\end{array}$ & 1,461 \\
\hline Dotcom Collapse & Apr. $10-14,2000$ & $-11.14 \%$ & $\begin{array}{l}\text { weekly return ending } \\
\text { with Apr. 7, } 2000\end{array}$ & 1,577 \\
\hline $\begin{array}{l}\text { U.S. Financial } \\
\text { Crisis }\end{array}$ & Oct. $6-10,2008$ & $-20.08 \%$ & $\begin{array}{l}\text { weekly return ending } \\
\text { with Oct. } 3,2008\end{array}$ & 1,578 \\
\hline \multicolumn{5}{|c|}{ Data for monthly returns } \\
\hline Crash & Date & Return & Data for forecasting & $\begin{array}{l}\text { Number of } \\
\text { observations }\end{array}$ \\
\hline Black Monday & Oct. 1987 & $-24.54 \%$ & $\begin{array}{l}\text { monthly return ending } \\
\text { with Sept. } 1987\end{array}$ & 332 \\
\hline $\begin{array}{l}\text { U.S. Financial } \\
\text { Crisis }\end{array}$ & Oct. 2008 & $-18.42 \%$ & $\begin{array}{l}\text { monthly return ending } \\
\text { with Sept. } 2008\end{array}$ & 359 \\
\hline
\end{tabular}




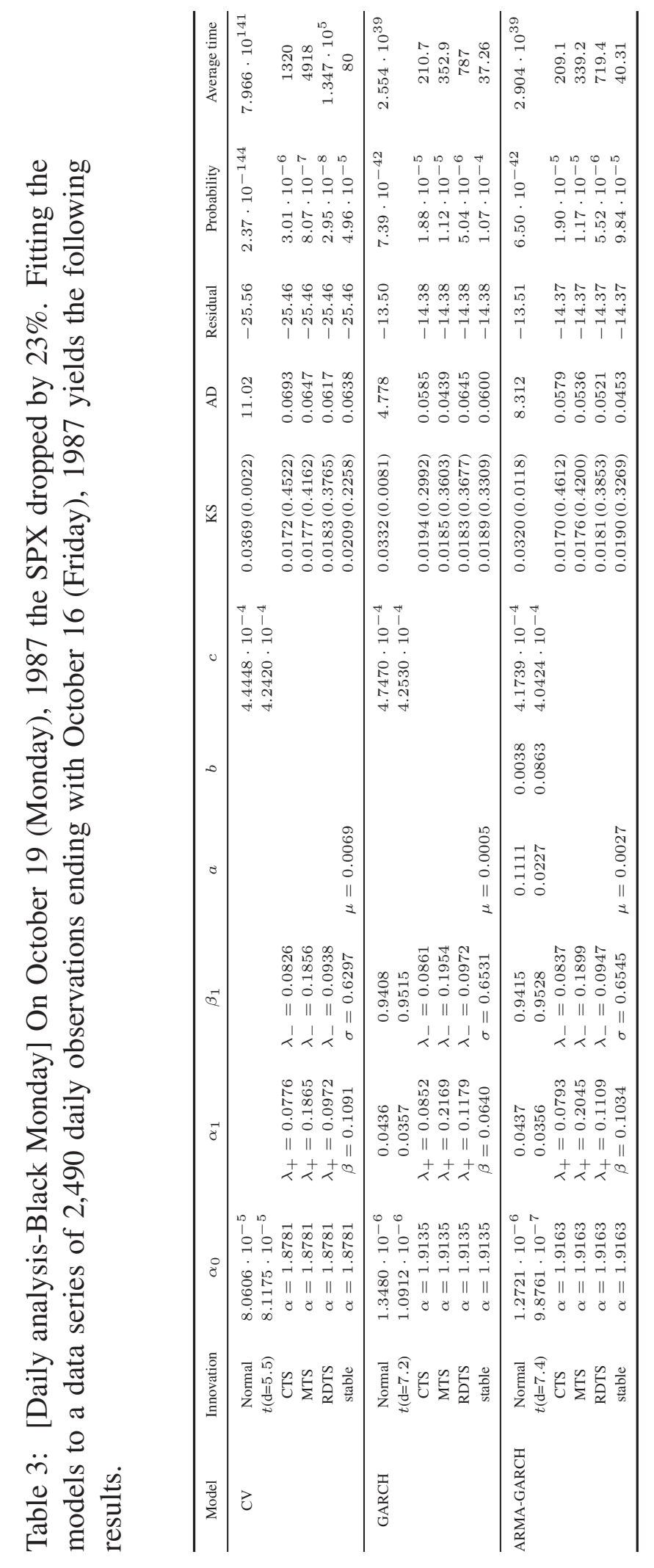




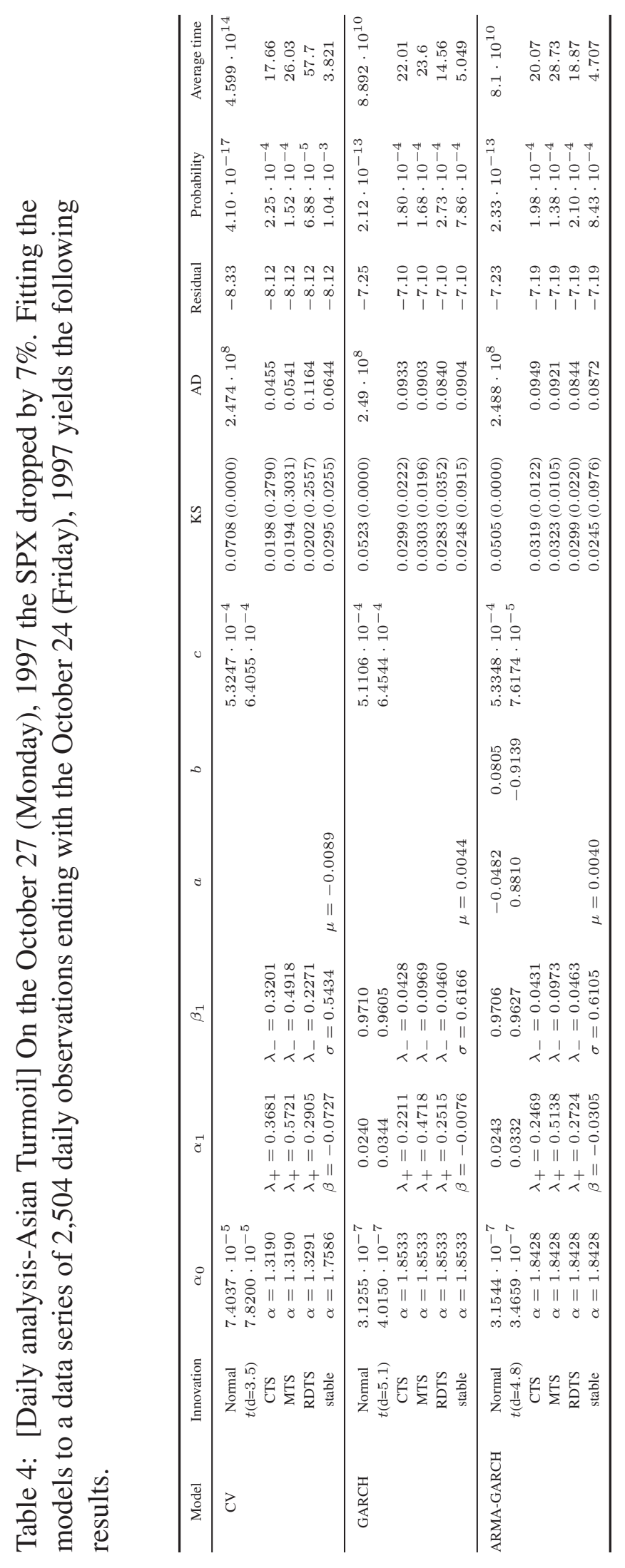




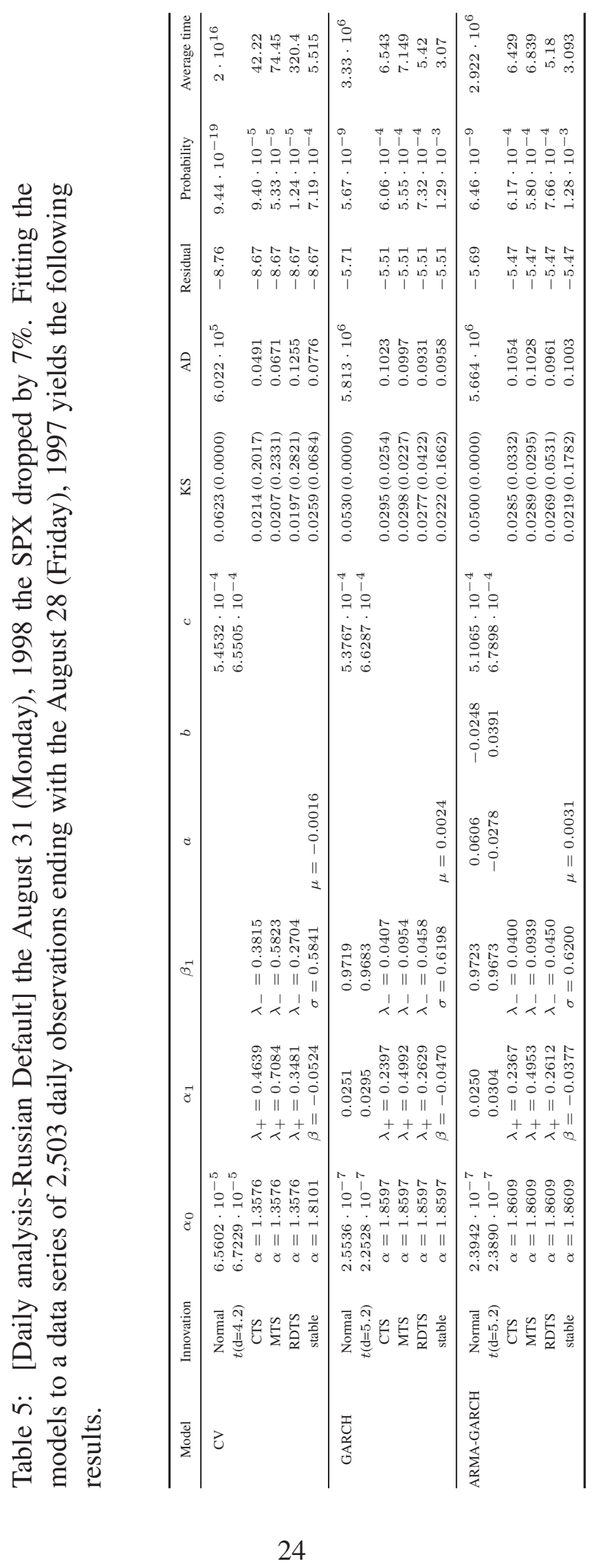




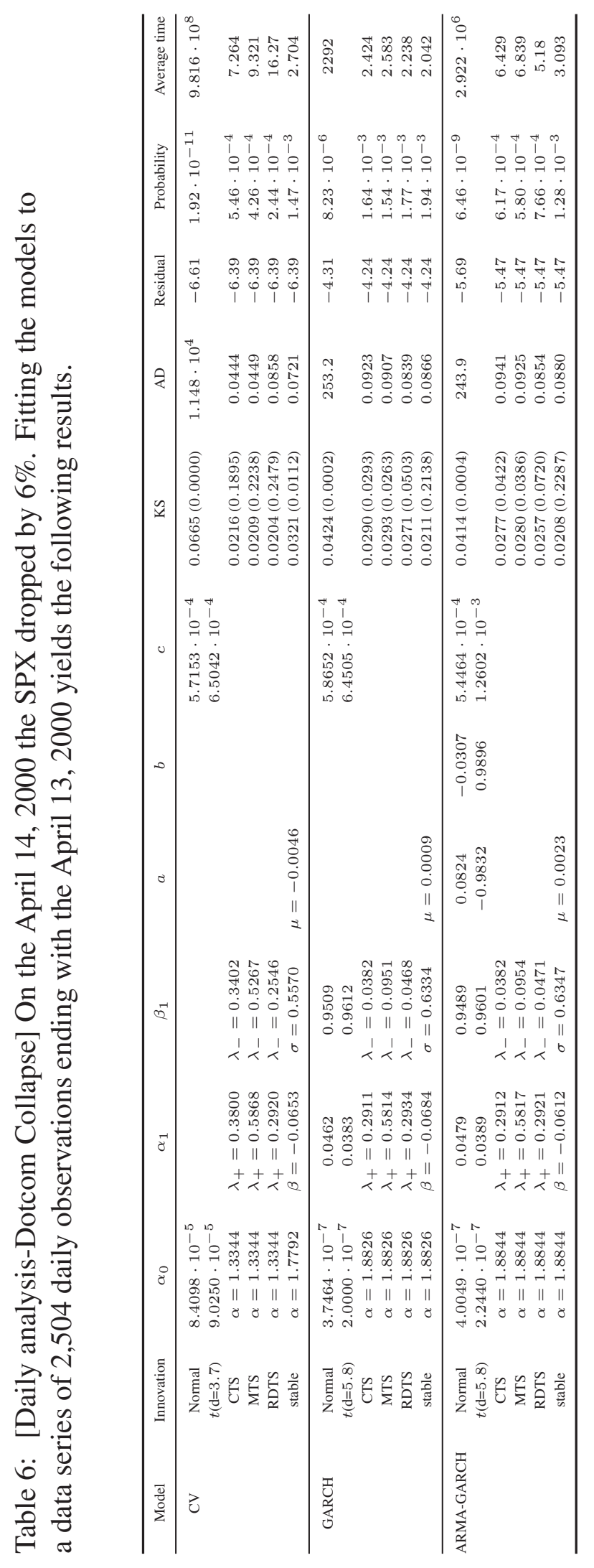




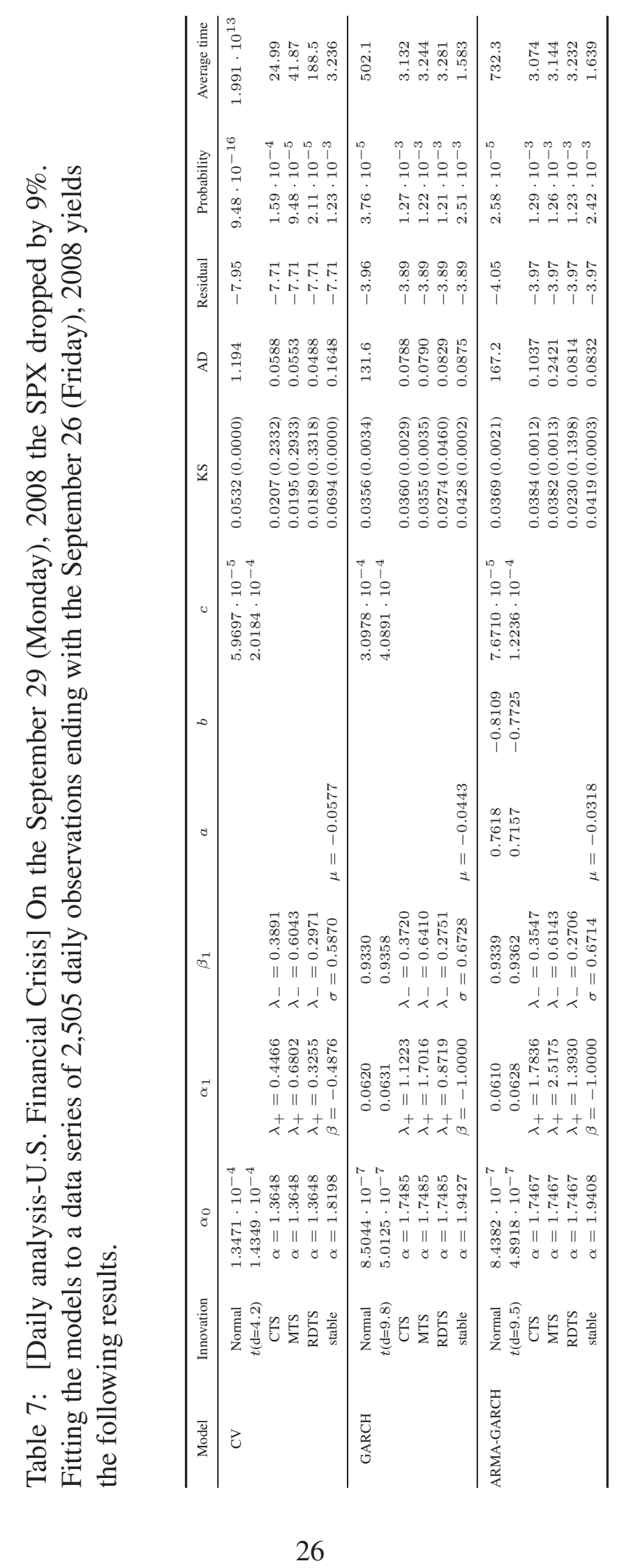




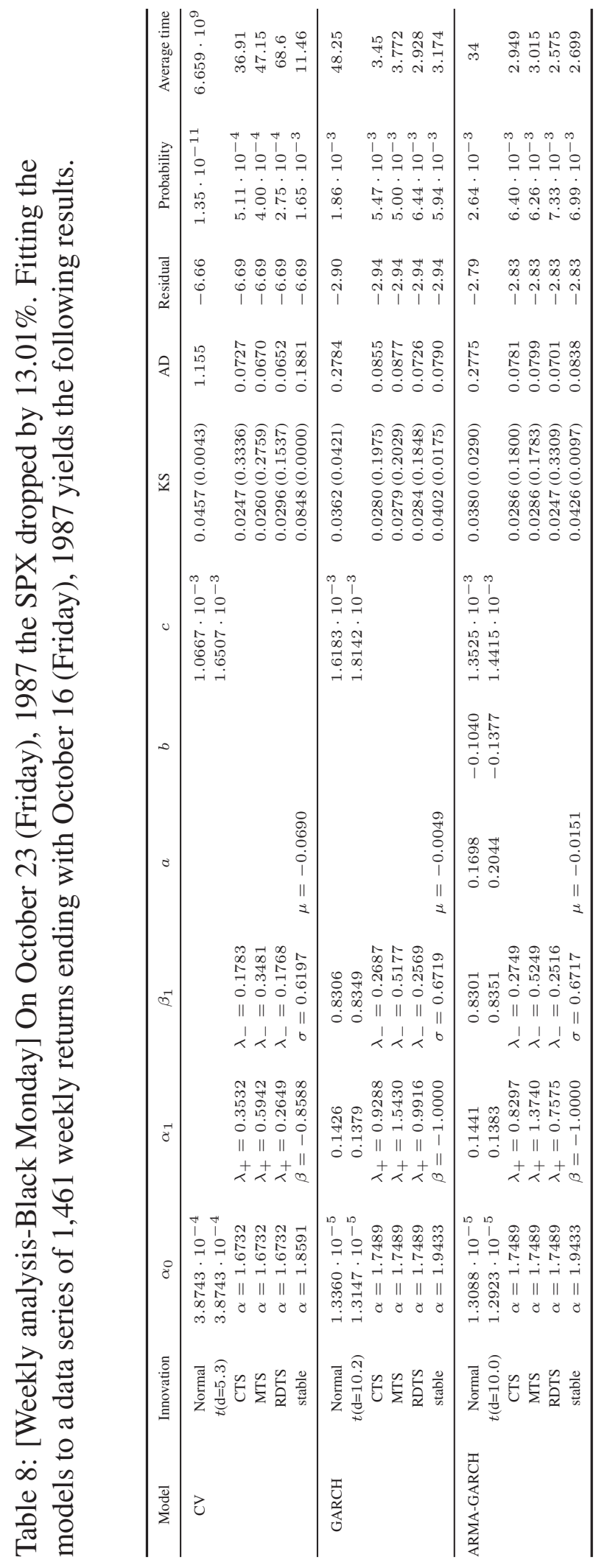




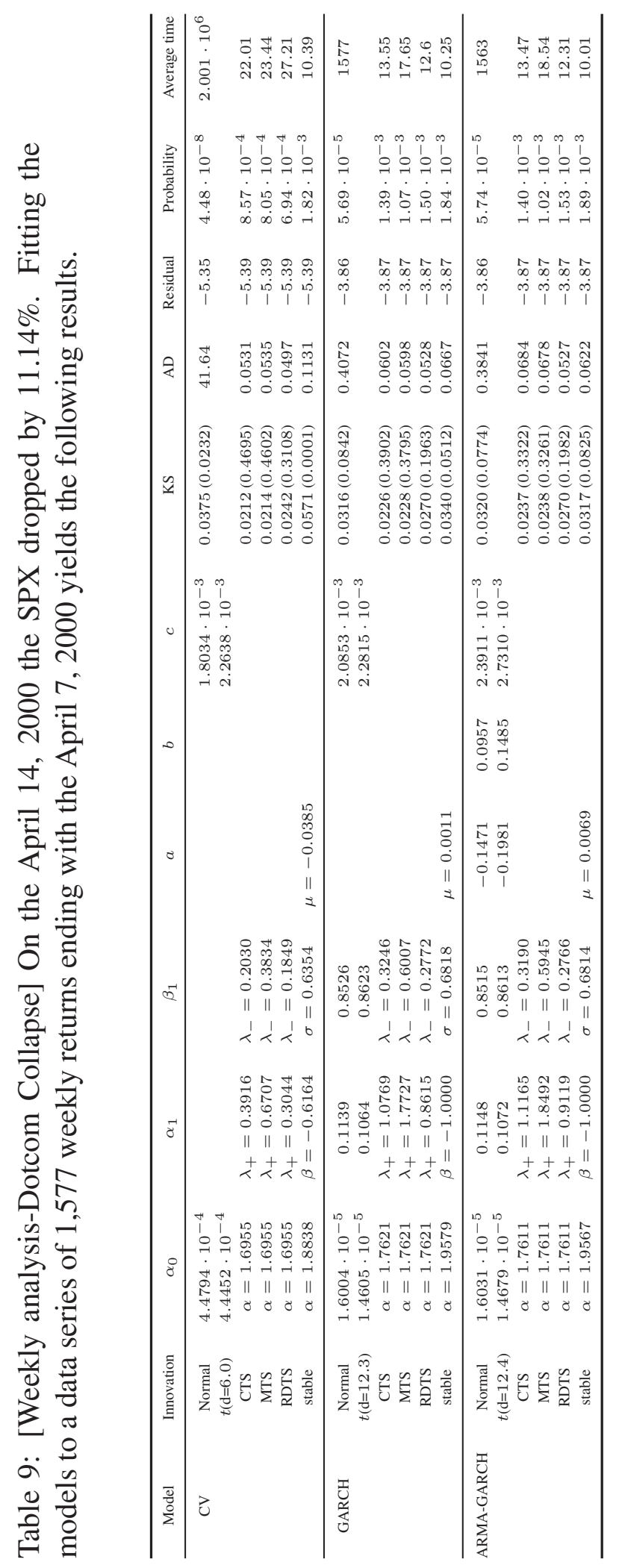




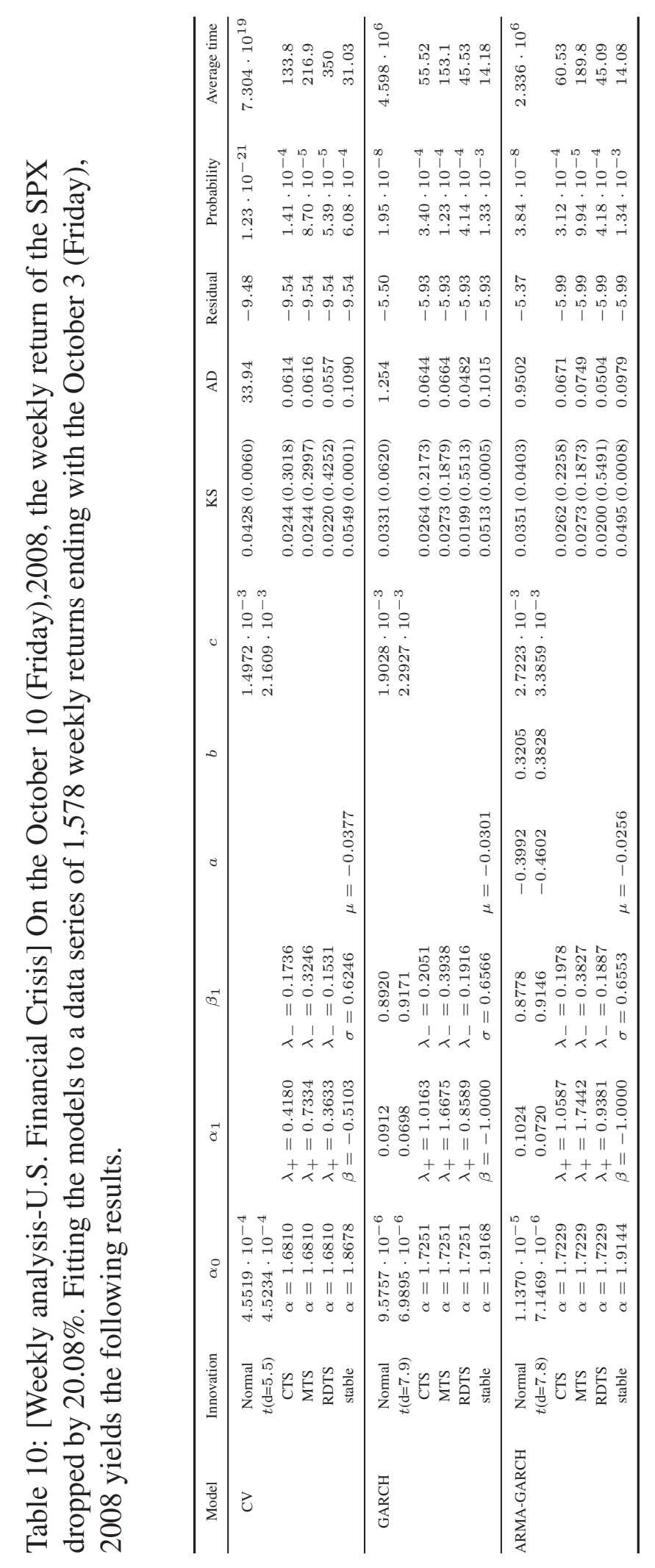




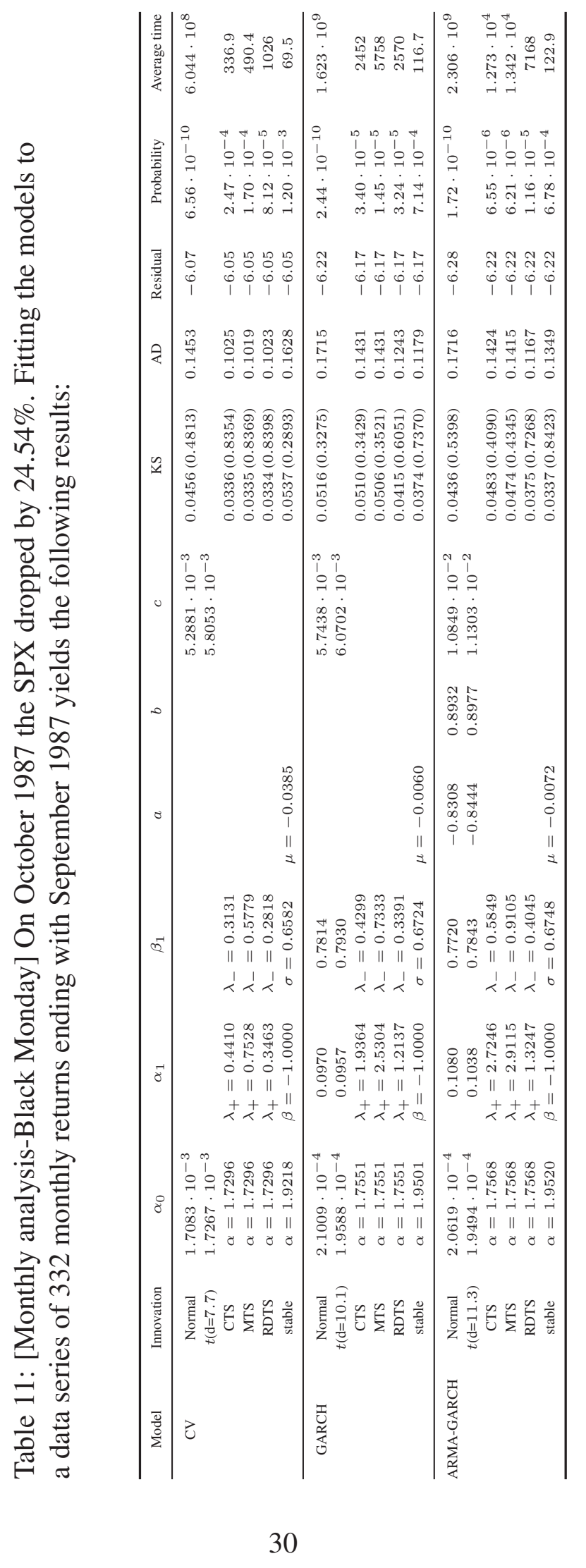




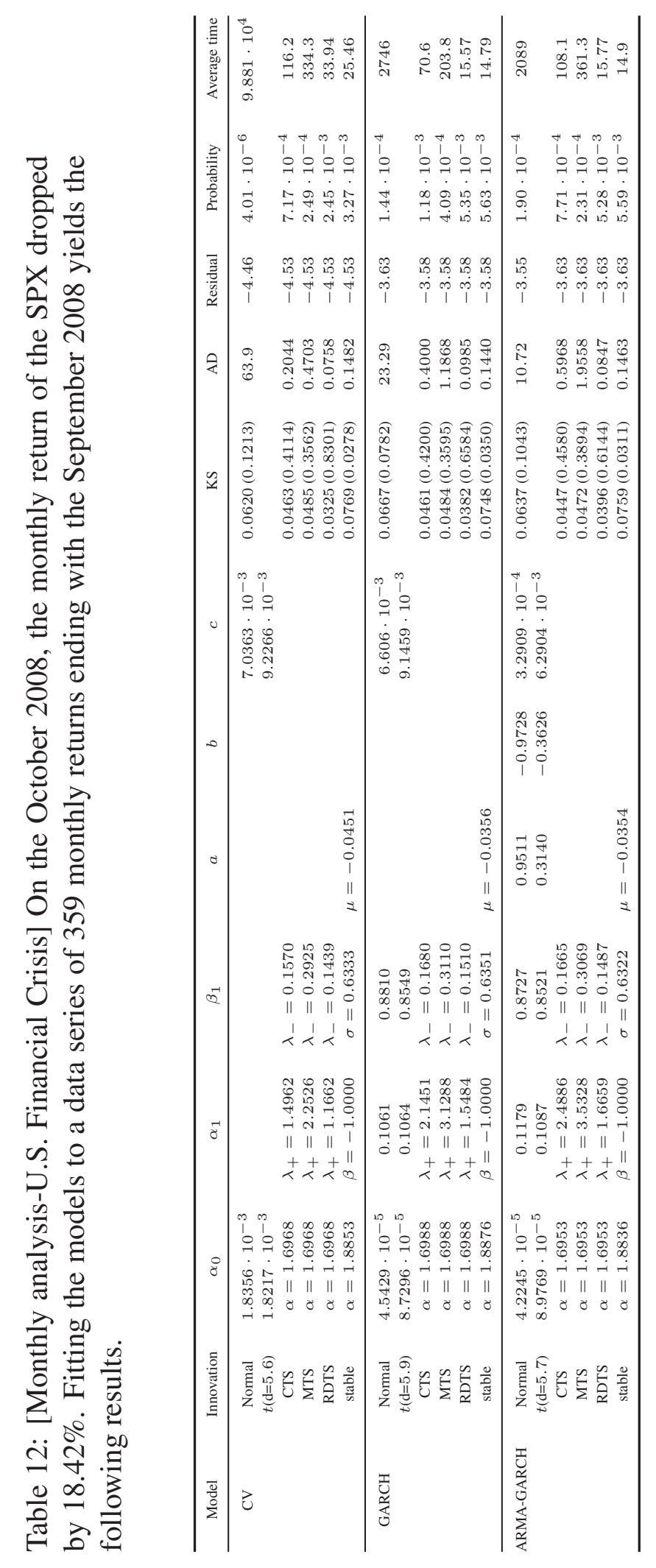




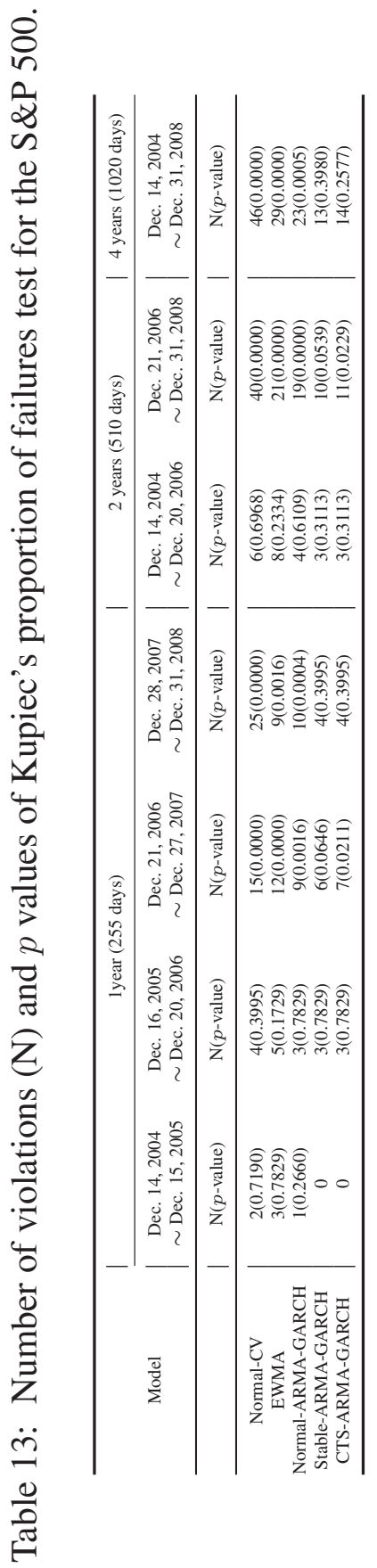



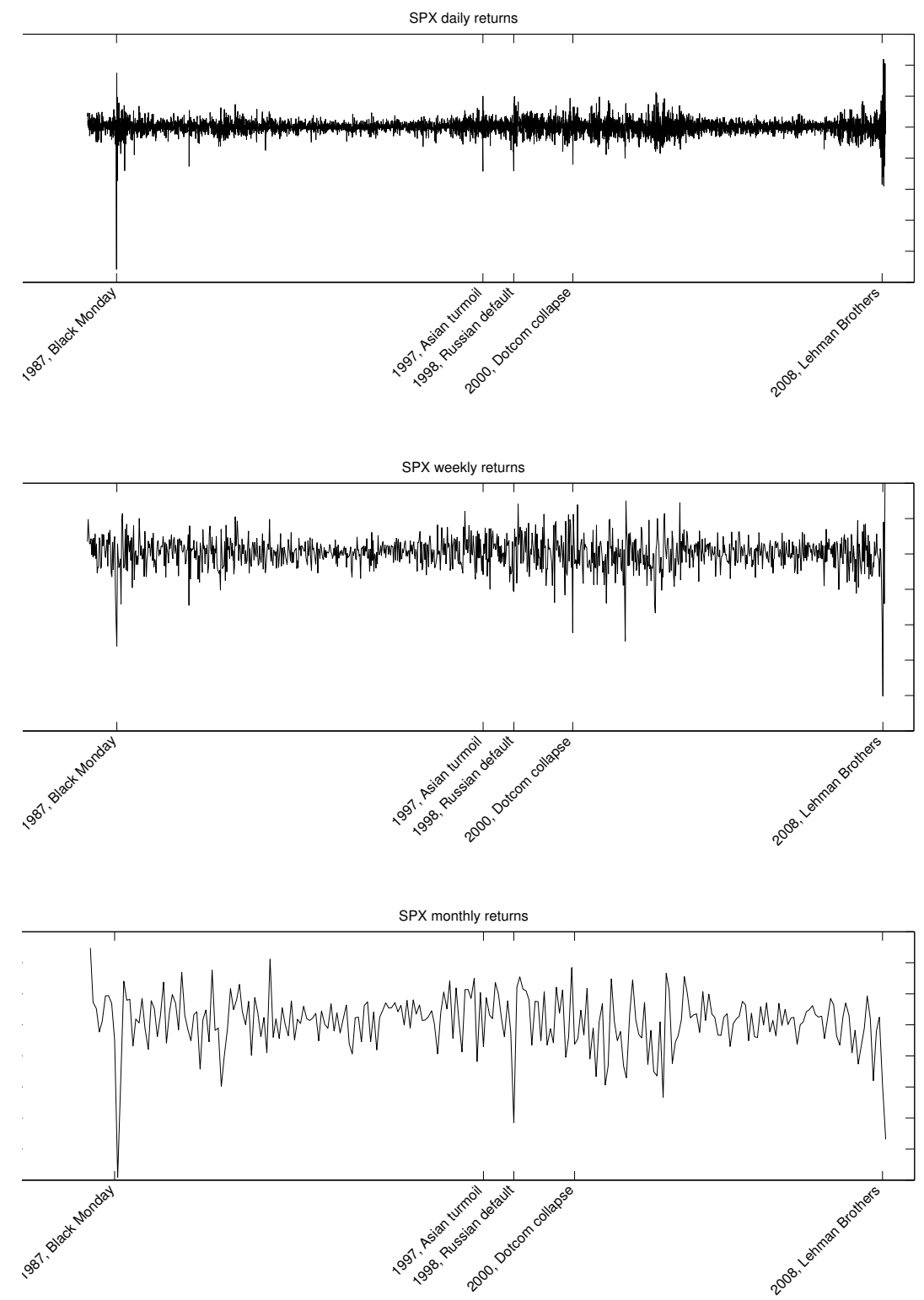

Figure 1: Daily, weekly, and monthly returns of the SPX from January 1987 to December 2008. 


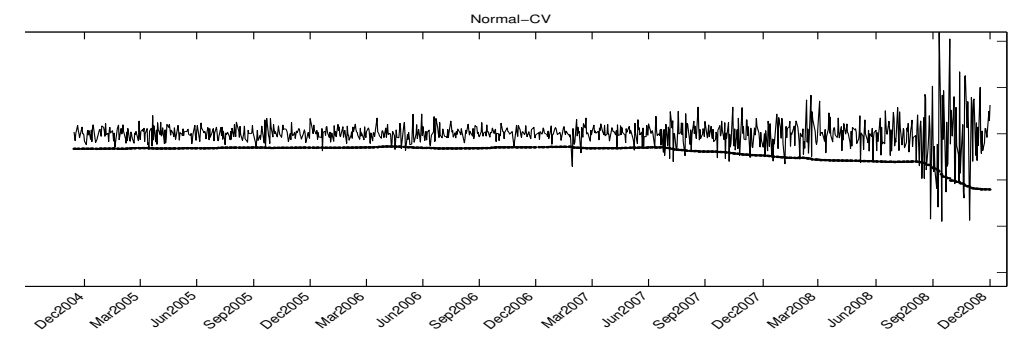

EWMA
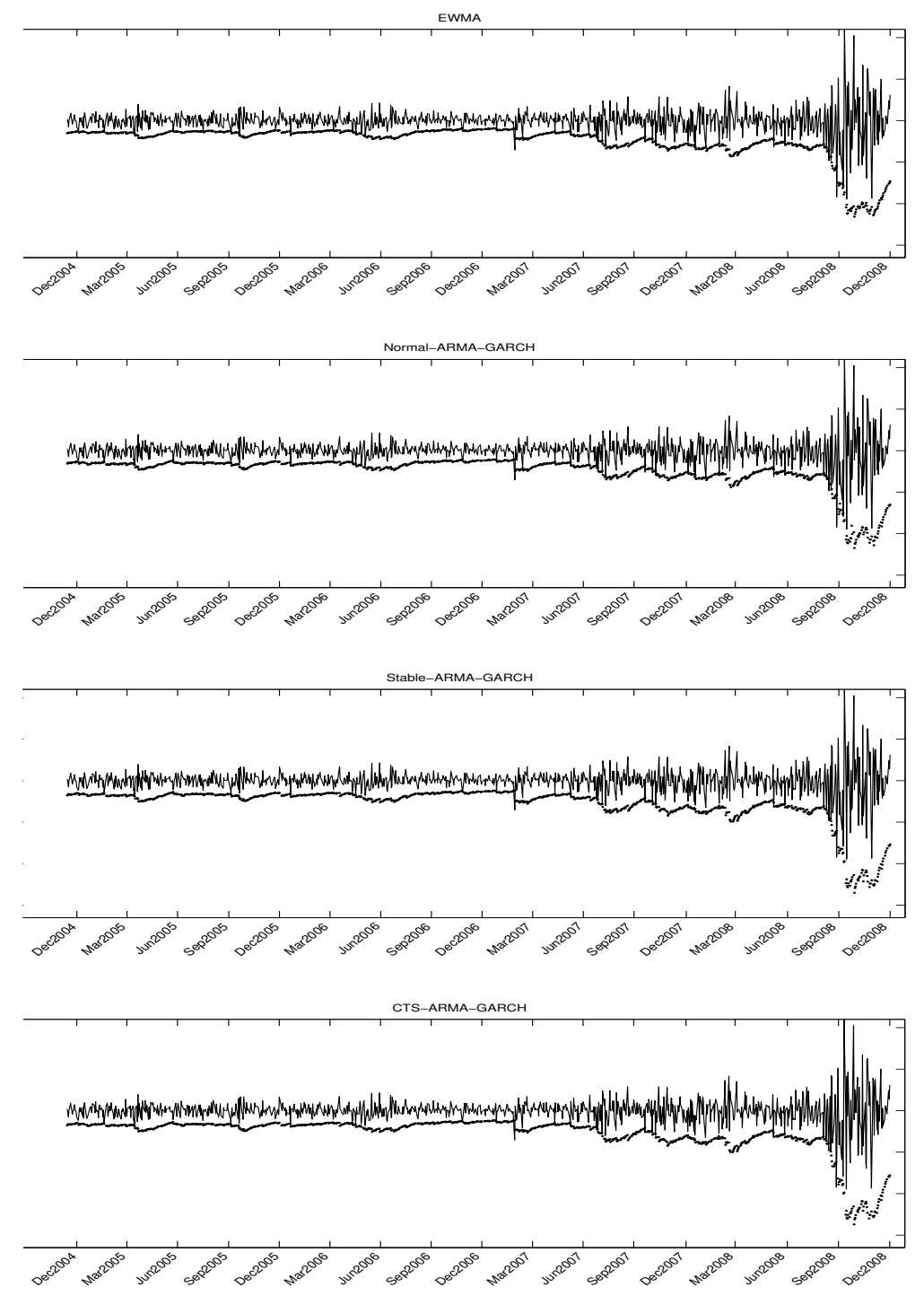

Figure 2: $\operatorname{VaR}\left(-\operatorname{VaR}_{t, \eta}\left(y_{t+1}\right)\right)$ for the SPX returns for the normal-CV, the EWMA, the normal-ARMA-GARCH, the stable-ARMA-GARCH, and the CTS-ARMA-GARCH model. 


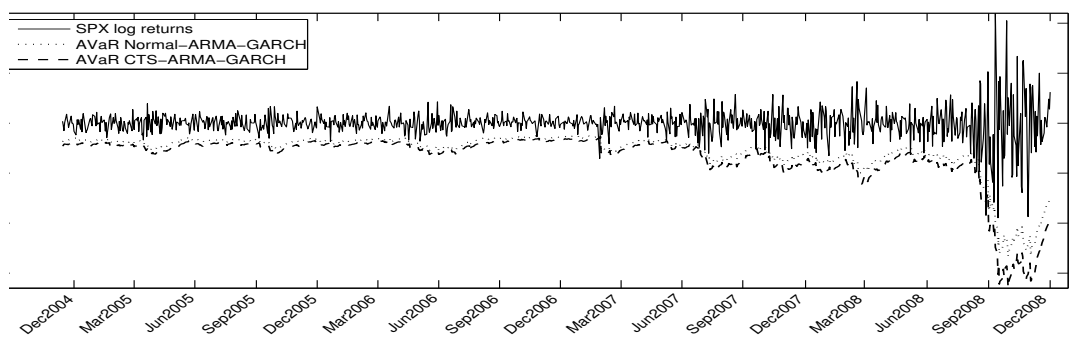

Figure 3: The 1\%-AVaRs $\left(-\operatorname{AVaR}_{t, 0.01}\left(y_{t+1}\right)\right)$ of the SPX, for the normal-ARMAGARCH and CTS-ARMA-GARCH models.

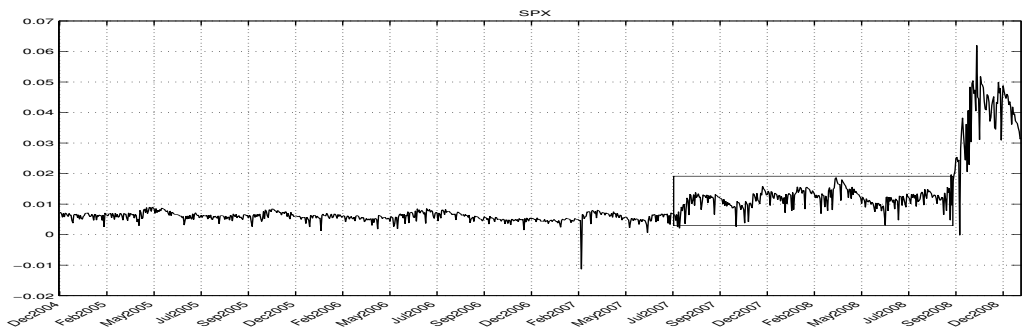

Figure 4: The differences between 1\%-VaR for the normal-ARMA-GARCH and 1\%AVaR for the CTS-ARMA-GARCH models with respect to the SPX. The differences are increasing in the solid rectangles.

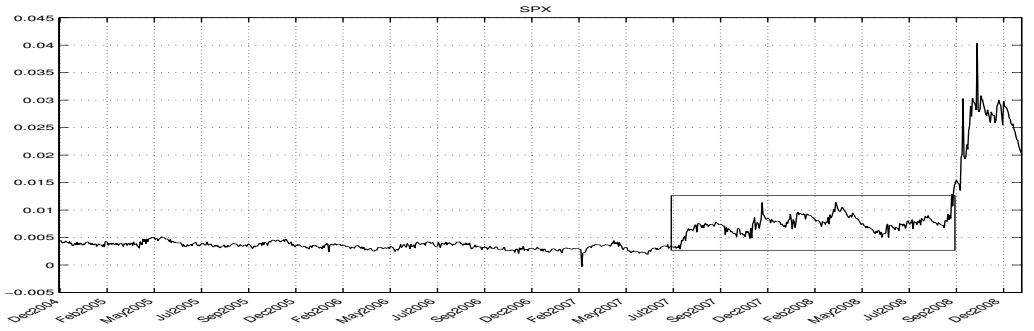

Figure 5: The differences between 1\%-AVaR for the normal-ARMA-GARCH and 1\%AVaR for the CTS-ARMA-GARCH models with respect to the SPX. The differences are increasing in the solid rectangles. 


\section{Working Paper Series in Economics}

recent issues

No. 2 Young Shin Kim, Svetlozar T. Rachev, Michele Leonardo Bianchi, Ivan Mitov, Frank J. Fabozzi: Time series analysis for financial market meltdowns, August 2010

No. 1 Biliana Güner, Svetlozar T. Rachev, Daniel Edelman, Frank J. Fabozzi: Bayesian inference for hedge funds with stable distribution of returns, August 2010 